\title{
La Securite Juridique Du Contribuable Face a L'execution Du Controle Fiscal En Droit Congolais
}

Menga Rene Yaosuwa

Coord. National de BECRN ONG

DOI: $10.36348 /$ sijll.2020.v03i03.004

| Received: 17.03.2020 | Accepted: 24.03.2020 | Published: 30.03.2020

*Corresponding author: Menga Rene Yaosuwa

\section{Abstract}

L'Administration Fiscale a pour rôle de mobiliser les ressources fiscales qui sont prévues par la Loi des Finances d'un Etat. Cette mobilisation se réalise par la mise en exécution, par l'Administration Fiscale, des actes législatifs et réglementaires régissant le prélèvement fiscal. Ces derniers, constituant la législation fiscale, prévoit non seulement des prérogatives de l'administration fiscale mais aussi des droits et des obligations fiscales pour les contribuables. Pour ce faire l'administration fiscale doit pouvoir réaliser le contrôle fiscal dans le stricte respect des procédures fiscales, prévues dans la loi $\mathrm{n}^{\circ} 004 / 2003$ du 13 mars 2003 telle que modifiée et complétée à ce jour, de façon à garantir un prélèvement juste de l'impôt et aussi d'entretenir des bonnes relations avec les contribuables. Etant donné que ces derniers doivent être considérés comme des partenaires et non des esclaves fiscales. Ainsi, nous pouvons affirmer ce qui suit: une administration qui vous simplifie la vie trouvera en face un contribuable citoyen ; une administration qui respecte les personnes et le droit trouvera en face un contribuable coopératif et une administration équitable trouvera en face un contribuable loyal.

Keywords: Sécurité Juridique, Contribuable, Exécution, Control Fiscal et Droit Congolais.

Copyright @ 2020: This is an open-access article distributed under the terms of the Creative Commons Attribution license which permits unrestricted use, distribution, and reproduction in any medium for non-commercial use (NonCommercial, or CC-BY-NC) provided the original author and source are credited.

\section{INTRODUCTION}

\section{Problematique}

L'Administration Fiscale a pour rôle de mobiliser les ressources fiscales qui sont prévues par la Loi des Finances d'un Etat. Cette mobilisation se réalise par la mise en exécution, par l'Administration Fiscale, des actes législatifs et réglementaires régissant le prélèvement fiscal. Ces derniers, constituant la législation fiscale, prévoit non seulement des prérogatives de l'administration fiscale mais aussi des droits et des obligations fiscales pour les contribuables.

En outre, elle est aussi un organe technique d'assistance du pouvoir exécutif, le Gouvernement, dans l'élaboration de la politique fiscale et des reformes structurelles et législatives.

Par Administration Fiscale, nous faisons allusions au Fisc, le service des Impôts, tel la Direction Générale des Impôts (DGI) en République Démocratique du Congo. C'est un service du Ministère ayant les finances dans ses attributions et est doté d'une autonomie administrative et financière. Ce service n'est pas le seul pourvoyeur de recettes de l'Etat, au niveau central, il y aussi la Direction Générale des Douanes et
Accises (DGDA) et al. Direction Générale des recettes administratives, domaniales, judiciaires et de participation (DGRAD).

Le système fiscal congolais est déclaratif, c'est-à-dire qu'il appartient au contribuable de déclarer lui-même à l'administration la matière sur laquelle sera prélevée l'impôt. Il s'agit donc d'une confession fiscale. Comme la confiance n'exclut pas le contrôle, le législateur a attribué à l'administration le pouvoir de contrôlé les déclarations souscrites par les redevables afin de vérifier les éléments justifiant l'établissement de l'impôt.

Le contrôle apparaît donc comme la contrepartie du système déclaratif. L'administration fiscale ne doit pas considérer ce qui a été déclaré comme absolu car le contribuable minore de temps en temps les bases d'impositions.

Bien que l'administration fiscale dispose des prérogatives de contrôle, le législateur a aussi prévu certaines dispositions fiscales qui assurent la protection $\mathrm{du}$ redevable contre tout abus de droit de l'administration en matière de contrôle. 
Cette protection, permettant de limiter tout excès ou débordement des agents de l'administration fiscale, constitue une garantie que le législateur accorde aux redevables. C'est ainsi que dans le cadre de cette étude, nous nous proposons d'analyser les garanties des contribuables face à la mise en œuvre du contrôle fiscal par la Direction Générale des impôts.

\section{HYPOTHESE}

Pour réaliser cette étude, nous fondons sur l'hypothèse selon laquelle que la mise en œuvre du contrôle fiscal par l'administration fiscale, à travers les vérificateurs des impôts, ne se réaliserait pas comme prévu par les dispositions légales en la matière. Les contribuables sont souvent victimes, du fait de l'ignorance de leurs droits, du terrorisme fiscal des agents du fisc commis au contrôle.

Du reste, les procédures permettant de mettre en œuvre les pouvoirs d'investigation et les pouvoirs de contrôle incombant à l'administration seraient différentes et le contribuable a, dans les deux hypothèses, des droits et des garanties qui délimitent les prérogatives administratives de contrôle.

A cet égard, le législateur soucieux de maintenir un certain équilibre entre les parties entendrait assortir les procédures de contrôle fiscal d'une série de garanties (sécurités), destinées à protéger les droits du contribuable contre les abus de l'administration notamment dans l'exercice de ses prérogatives de contrôle.

\section{INTERET DU SUJET}

L'intérêt de la présente étude est évident. Il est à la fois théorique et pratique. Sur le plan théorique, cette étude permettra au lecteur de comprendre les mécanismes d'exécution du contrôle fiscal par l'administration ainsi que les droits et les obligations des contribuables en matière de contrôle.

Sur le plan pratique, cet article constitue un outil de référence pour les contribuables, victimes des abus de l'administration fiscale dans l'exécution du contrôle, pour faire prévaloir leurs droits.

\section{CADRE METHODOLOGIQUE}

Pour réaliser cette étude, il nous a plu de recourir aux méthodes appropriées à savoir: la méthode juridique et la méthode sociologique.

La méthode juridique nous a permis de comprendre la portée des instruments juridiques relatifs à l'objet sous examen.

Cette méthode a été complétée utilement par la méthode sociologique laquelle repose sur l'étude des faits sociaux qui entrent en relation avec le phénomène observé. Elle nous permet de comprendre les illustrations évoquées en tant que faits sociaux, leurs appréhensions et leurs intégrations dans l'évolution de la société, particulièrement la société fiscale congolaise.

\section{Ces méthodes ont été appuyées par les techniques suivantes}

La technique documentaire, cette technique trouve ici son sens du fait qu'elle nous a servi dans l'exploitation des mémoires, thèse, ouvrages et autres documents ayant trait à notre étude;

La technique de l'interview, qui va de pair avec la précédente pour des raisons de complément d'information. Elle nous a permis de collecter certaines données lors des communications verbales avec les responsables du Centre des Impôts.

\section{DELIMITATION DE L'ETUDE}

Cette étude se limitera à l'analyse des dispositions régissant le contrôle fiscal en République Démocratique du Congo, prévues par la Loi $\mathrm{n}^{\circ}$ 004/2003 portant reformes des procédures fiscales telle que modifiée et complétée à ce jour.

\section{LES PROCEDURES DE CONTROLE FISCAL Notions sur le contrôle fiscal}

Le système fiscal congolais est déclaratif et auto-liquidatif. Le contribuable a l'obligation à déclarer les éléments servant de calcul des impôts auxquels il est assujettit et s'acquitter du paiement desdits impôts.

En contrepartie, la loi reconnait à l'Administration fiscale, le pouvoir de procéder au contrôle de la déclaration souscrite dont la véracité doit être prouvée par le contribuable (Code des impôts art, 25, 26, 27,28 de la loi ${ }^{\circ} 004 / 2003$ du 13 mars 2003 portant reforme des procédures fiscales telle que modifiée et complétée à ce jour).

Ainsi, le contrôle fiscal recourt aux techniques classiques et sauvegarde non seulement les intérêts du trésor mais également ceux de l'entreprise en la poussant à une meilleure gestion pour éviter des pénalités sur des éventuelles tricheries. Il sied de rappeler que tout contrôle effectué par n'importe quel organe est toujours mal perçu par l'entité contrôlée qui se doute toujours de son issue.

Les éléments souscrits par les contribuables à l'Administration fiscale sont minutieusement examinés et sur le plan formel et sur celui du fond. Pour ce faire, la Direction Générale des Impôts utilise diverses techniques, dispositions légales et procédures pour déceler des fraudes, des erreurs, des omissions des tricheries en vue d'asseoir des impôts à mettre à charge du contribuable et dont le recouvrement sera effectué sans contestation. 
Cependant les contribuables n'acceptent pas de gaieté de cœur le contrôle fiscal initié par la DGI car ils s'attendent toujours au décaissement supplémentaire des fonds au profit du Trésor Public.

Le contrôle fiscal doit être à cet effet, un outil conciliateur des points de vue divergents de l'Administration Fiscale et des contribuables, capable d'asseoir des impôts justes ne pouvant pas souffrir d'une quelconque contestation pour permettre un meilleur rendement des services mobilisateurs des recettes du Trésor public.

\section{L'importance du contrôle fiscal}

Au fil des années, le contrôle a été amélioré par diverses méthodes et procédures qui ont poussé des entreprises à mieux tenir leurs comptabilités et à accorder une grande importance au contrôle de leur gestion.

L'importance croissante de la nécessité d'un contrôle interne a amené les entreprises à mettre en place des services chargés de vérifier l'existence et la bonne application des contrôles comptables, financiers et opérationnels, de contrôler la conformité des titres ( actions ) et des comptes avec les plans, les politiques et les procédures déterminés par leurs responsables décideurs en conformité avec les règles juridiques et fiscales déterminées par le pouvoir public.

Les divers responsables des sociétés édictent des règles susceptibles d'améliorer le fonctionnement de leurs entreprises. Ainsi, le contrôle aide à déceler les irrégularités dans la gestion, à déterminer les erreurs et les omissions, relevées à divers niveaux d'une entreprise. Le contrôle est de ce fait une nécessité économique et sociale.

La nécessité économique du contrôle se caractérise par l'attention particulière que les dirigeants d'une société accordent au respect de divers principes de gestion en vue de la réalisation des profits tandis que l'importance sociale du contrôle est déterminée par la recherche de la paix sociale au sein de l'entreprise sur base de la vérification des taux des salaires des employés et le versement régulier des rémunérations à ces derniers.

En définitive, le contrôle permet à toute entreprise de mieux maitriser les paramètres inhérents à l'environnement économique dans lequel elle évolue. L'entreprise utilisera pour ce faire diverses techniques pour une meilleure gestion notamment la comptabilité analytique d'exploitation, la gestion budgétaire, l'audit interne, la comptabilité générale, etc.

\section{L'ORGANISATION DU CONTROLE FISCAL Le contrôle formel}

Par le contrôle formel, la DGI examine minutieusement la forme et le fond de tous les éléments déclarés de manière à ce que toutes les données soient correctement saisies au niveau du service informatique.

Ce contrôle porte sur tous les documents déposés par le contribuable à la DGI et permet un traitement efficace de toutes les informations et aide à éviter des impositions incorrectes.

\section{Le contrôle sur pièces}

Le contrôle sur pièce est une vérification, effectuée au niveau du bureau, de la situation fiscale et de tous les documents constitutifs du dossier unique du contribuable.

Il a pour rôle de découvrir des erreurs, des insuffisances, des omissions ou des dissimulations et la recherche des incohérences en vue d'asseoir des impositions.

Ce contrôle amène le vérificateur à déterminer les pistes de ses recherches susceptibles de l'aider à mieux orienter la vérification- (DGI : Guide du contrôle sur pièce Déc.2006). Il est à préciser qu'à l'issue du contrôle sur pièces, des demandes des éclaircissements ou des justifications peuvent être adressées aux contribuables et des notifications des impôts également.

Toutefois, le contrôle sur pièces n'est efficace que s'il est effectué sur trois ou quatre ans successifs en vue de mener une étude comparative de divers éléments de la comptabilité du contribuable.

\section{PROCEDURES DE CONTROLE FISCAL}

Les déclarations souscrites par les contribuables sont relatives aux opérations effectuées pendant l'exercice comptable allant du $1^{\mathrm{e}}$ janvier au 31 décembre.

Cependant, la DGI, sur base de la loi, peu procéder au contrôle des revenus des exercices non encore contrôlés et ce conformément au principe du droit de rappel (de cinq ans) déterminé par la loi n004/2003 du 13 mars 2003 telle que modifiée a ce jour en son article 43 . Il est précisé qu'à l'issue du contrôle, l'Administration fiscale peut rectifier les déclarations inexactes souscrites, lorsque les erreurs ou amissions sont jugées graves.

L'Administration fiscale sélectionne les entreprises à contrôler afin d'éviter une vérification systématique de toutes les sociétés.

Le vérificateur des impôts, muni d'un ordre de vérification signé par le fonctionnaire compétent, adresse un avis de vérification au contribuable, au moins huit jours avant la date de la première intervention. 
Cet avis informe le redevable notamment de son droit de se faire assister d'un conseil de son choix et précise la nature des impôts ou autres droits ainsi que la période soumise au contrôle-(Code des impôts mis à jour au 30/09/2003 article 28 et 30 de la loi nº04/2003 du 13 mars 2003 portant reforme des procédures fiscales telle que modifiée et complétée a ce jour).

L'attention des vérificateurs est attirée sur le respect de l'objet et la durée de la mission. La prorogation de cette durée ne peut être décidée que par le fonctionnaire signataire de l'ordre de vérification.

\section{LES OPERATIONS PRE CONTROLE La recherche et le recoupement}

La recherche et le recoupement est une opération qui consiste pour le vérificateur à rechercher auprès des tiers partenaires de l'entreprise (service publics, fournisseurs, banquiers chiens) des informations susceptibles de contredire les éléments souscrits par le contribuable sous contrôle. La demande des renseignements auprès des tiers est dévolue à tout agent de l'Administration des impôts- (La loi $\mathrm{n}^{\circ} 004 / 2003 \mathrm{du} 13$ Mars 2003 telle que modifiée et complétée a ce jour portant réforme des procédures fiscales art 46, 47, 48, 49, 50, 51, 52).

\section{L'enquête}

Par l'enquête, souvent effectuée par les inspecteurs des impôts, l'Administration fiscale cherche à réunir des informations à caractère général ou ponctuel en vue de faire aboutir une procédure de l'assiette, du contrôle, du contentieux ou du recouvrement-(La loi n004/2003 du 13 Mars 2003 portant réforme des procédures fiscales telle que modifiée et complétée a ce jour art 37).

\section{L'étude du dossier unique}

C'est un travail préparatoire du contrôle fiscal. Dénommée également? contrôle fur pièces", l'étude du dossier unique aide le vérificateur à rechercher, avant de descendre dans l'entreprise, des pistes intéressantes susceptibles de lui faciliter l'orientation du contrôle fiscal.

Pour ce faire, le vérificateur s'emploiera à dresser un tableau comparatif des derniers bilans. Plus ce tableau comprend plus d'exercices à comparer (au moins quatre), plus des renseignements sont éloquents.

En outre, le contrôleur s'attardera sur toutes les additions dans toutes les colonnes du bilan et du tableau de formation de résultat; il fera l'analyse critique des différents postes que comporte le bilan présenté par l'entreprise.

\section{L'aperçu de l'entreprise}

Le vérificateur ne peut commencer ses missions qu'après avoir visité l'entreprise et eu un entretien substantiel avec la Direction de l'entreprise et avec le Chef Comptable. Il doit savoir, entre autres, de façon précise à qui il peut s'adresser pour toutes informations et précisions qui lui seront nécessaires. L'entretien avec la Direction sera suivi d'une visite de l'exploitation en vue d'acquérir par la même occasion une notion exacte de l'appareil technique et administratif. Ce serait malencontreux de vouloir mener à bien un contrôle efficient sans connaitre la technique de l'entreprise que l'on a pour mission de contrôler.

\section{Les procedures de redressement et de notification}

Tel que déterminé dans les pages précédentes, l'Administration fiscale, sur base de la loi, procède au contrôle des tous les éléments déposés par le contribuable en vue de s'assurer de leur sincérité et régularité. Elle utilisera diverses techniques pour déceler des erreurs, des omissions, des irrégularités, des fraudes qui l'amèneront à déterminer les suppléments d'impôts à mettre à charge des contribuables fautifs.

Le contrôle fiscal revêt donc un caractère technique qui exige un vaste ensemble de connaissances comptables et fiscales de la part du vérificateur des impôts qui, à la clôture de sa mission doit engager un débat contradictoire avec le comptable ou le conseil de l'entreprise sur tous les points qu'il a retenus pour déterminer le suppléments d'impôts.

\section{Securite juridique des contribuables face a l'execution du controle fiscal \\ Les garanties insuffisantes dans la phase de contrôle \\ Pour constater les obligations fiscales du contribuable dans le cadre du système déclaratif, l'administration tient de la loi deux prérogatives de contrôle: le pouvoir d'investigation et le pouvoir de vérification.}

Par ailleurs, le droit pour le contribuable d'être informé constitue une garantie essentielle des droits de la défense-(Manuel des procédures fiscales de novembre 2004 DGI RDC p.47 1èr §).

L'information est souvent une condition préalable à l'application effective d'autres garanties offertes par la loi, notamment quand l'administration est amenée à exercer son droit de contrôle à l'encontre du contribuable.

Ce droit à l'information implique alors que le contribuable soit préalablement averti des mesures qui le concernent. Toutefois, cette obligation d'informer le contribuable mise à la charge de l'administration ne se pose pas toujours, lorsque les mesures diligentées ne sont pas de nature à influer immédiatement sur les intérêts propres du contribuable.

Tel est notamment le cas, lorsque l'administration exerce son droit de communication en vue de recueillir des informations supplémentaires sur la situation fiscale du contribuable vérifié, du moins 
tant qu'elle n'oppose pas ces informations au contribuable lui même.

En outre, le non respect de l'obligation d'information $\mathrm{du}$ contribuable trouve encore à s'expliquer, particulièrement dans les cas où l'administration est amenée à exercer certaines procédures d'investigation considérées comme contraignantes, où l'information du contribuable ôterait tout intérêt à la procédure mise en œuvre. C'est le cas du contrôle dit « inopiné », qui a pour but d'effectuer des constatations purement matérielles. Ce type de contrôle a vocation à s'appliquer en particulier aux contribuables astreints à la tenue d'une comptabilité.

Ainsi, l'administration a désormais la possibilité d'engager à l'encontre de cette catégorie de contribuables le contrôle «inopiné » qui doit toutefois se limiter à la constatation matérielle des éléments physiques de l'exploitation, de l'existence et de l'état des documents comptables.

Hormis ces situations particulières qui justifient donc l'absence d'information préalable du contribuable, ce dernier doit en principe être avisé avant le début d'une opération de contrôle ou la mise en œuvre d'une procédure à son encontre. Ce principe est d'application générale malgré l'absence de procédure contradictoire, y compris dans les hypothèses, où les droits de la défense sont réduits à leur plus simple expression.

La portée de la garantie liée à l'obligation d'information du contribuable dans le cadre d'une vérification de comptabilité

L'administration supporte une obligation d'informer le contribuable de l'origine et de la teneur des renseignements obtenus par son droit de communication, afin de lui permettre de les contester dans le respect du contradictoire.

La notion de contradictoire doit ici être entendue dans un sens restrictif, dans la mesure où l'administration peut très bien mettre en œuvre son droit de communication sans en informer le contribuable et donc sans engager la moindre discussion contradictoire avec lui.

Mais, si l'administration n'a pas l'obligation d'engager un débat contradictoire avec la personne soumise au droit de communication dans les conditions évoquées, elle ne peut en revanche utiliser les informations obtenues à l'encontre du contribuable.

Ceci implique que le contribuable soit suffisamment informé de la nature et de la teneur des renseignements recueillis et qu'il soit à même d'en demander la communication et de les contester.
Il apparaît ainsi que l'information du contribuable est obligatoire et doit être formalisée dans la notification de redressement dans le cadre de la procédure contradictoire. La notification de redressement constate alors les conditions d'obtention des renseignements recueillis, en précisant notamment la procédure d'investigation ayant permis de les obtenir et l'identité du tiers auprès duquel cette procédure a été diligentée.

Du reste, le pouvoir de communication permet à l'administration de recouper les renseignements recueillis et donc de contrôler les déclarations du contribuable soumis à contrôle fiscal. Précisons ici que le renseignement susceptible d'être utilisé par l'administration peut concerner notamment un taux de marge commerciale du secteur d'activité où opère le contribuable, que celle-ci va lui opposer pour asseoir le redressement fiscal dans le cadre d'une procédure de reconstitution du chiffre d'affaires.

\section{Les garanties du contribuable soumis à une} procédure de vérification de comptabilité

Le droit pour le contribuable d'être informé constitue une garantie essentielle des droits de la défense dans le contexte d'une procédure de contrôle diligentée par l'administration. L'information est souvent une condition préalable à l'application effective d'autres garanties offertes par la loi.

Ainsi, cette information se concrétise en présence d'une vérification de comptabilité par la remise d'un avis de vérification au contribuable vérifié (Code des impôts op.cit, page 33).

Elle se traduit aussi par la mention faite au contribuable qu'il dispose de la faculté de se faire assister d'un conseil de son choix et par la notification formelle des résultats du contrôle. Les garanties ainsi instituées interviennent s'agissant des vérifications de comptabilité avant la vérification, pendant son déroulement et après son achèvement.

Nous nous proposons ici d'examiner successivement les garanties du contribuable préalables à la mise en œuvre de la vérification, celles à respecter pendant son déroulement et enfin celles liées son achèvement.

\section{Les garanties attachées à la procédure de redressement contradictoire}

Lorsque le service des impôts envisage d'apporter des rectifications aux bases d'imposition initiales, notamment dans le cadre d'une procédure de redressement contradictoire, il doit en premier lieu adresser au contribuable vérifié une notification de redressement. La procédure de redressement contradictoire débute alors par une notification de redressement et se poursuit par la possibilité donnée au contribuable de présenter ses observations. 
Le caractère contradictoire de la procédure se matérialise non seulement par les observations formulées par le contribuable sur les redressements proposés, mais encore par l'obligation faite à l'administration de répondre de façon motivée à ces observations.

Le caractère contradictoire de la procédure permet enfin au contribuable, soumis à une vérification de comptabilité, de saisir les commissions de taxation prévues aux articles 104 et 108 du Code des impôts livre deuxième préalablement à la mise en recouvrement des impositions.

Il apparaît ainsi que la procédure de redressement contradictoire est organisée autour de cette formalité substantielle, à savoir la notification de redressement. Mais encore il y a lieu de constater que l'exercice de cette prérogative de redressement est encadré strictement par le législateur permettant de ce fait d'assortir la procédure de redressement de garanties destinées à faire respecter les droits de la défense.

Les dispositions du code général des impôts ne définissent pas les formes que doit revêtir la notification de redressement. Toutefois, l'article 37 du Code des impôts livre deuxième en traitant de la procédure contradictoire de redressement pose la nécessité de cette formalité substantielle qu'est la notification de redressement : la notification est l'expression d'une décision et le premier acte de procédure.

La procédure de redressement est en principe contradictoire dès lors que le contribuable contrôlé a accompli ses obligations déclaratives. Ceci se traduit par le fait que le contribuable dispose d'un droit de réponse dont l'exercice est garanti par l'octroi d'un délai suffisant. La notification ouvrant alors un droit de réponse au contribuable, enserré toutefois dans le délai de vingt jours dans la procédure contradictoire, Celui-ci peut soit accepter le redressement formellement ou tacitement, soit présenter des observations. On peut donc envisager des conséquences différentes selon la réponse donnée par le contribuable.

Les droits des contribuables face au pouvoir de sanction en matiere de controle fiscal par l'administration

Comme nous l'avons évoqué, l'administration dispose de la faculté de mettre en œuvre son droit de contrôle dans le système déclaratif en exerçant deux prérogatives de contrôle : le pouvoir d'investigation et le pouvoir de vérification.

Le pouvoir d'investigation confère à l'administration le droit de se faire communiquer certains documents détenus par des tiers-(Chapitre II du code des impôts). Les renseignements recueillis peuvent être utilisés sous certaines conditions à l'encontre du contribuable vérifié et permettent alors de recouper ses déclarations. La doctrine range traditionnellement, au titre des pouvoirs d'investigation de l'administration, le droit de communication.

A cet égard, il importe de constater que le droit de communication apparaît de plus en plus comme l'instrument de portée générale sur lequel l'administration fonde l'essentiel de ses pouvoirs de contrôle.

Il a un domaine d'application très large et, est systématiquement utilisé en matière de contrôle des impôts déclaratifs, tels l'impôt sur les bénéfices des sociétés ou encore la taxe sur la valeur ajoutée. Il est distinct du pouvoir de vérification. Celui-ci répond au souci d'une analyse très fine de la matière imposable, notamment en matière de vérification de comptabilité. Cependant, nous allons voir que nous pouvons rattacher également, au titre des pouvoirs d'investigation de l'administration, le contrôle "inopiné "- (art 31de l'ordonnance loi $\mathrm{n}^{\circ} 13 / 005$ du 23 février 2013 telle que modifiée et complétée a ce jour) appeler en d'autre terme droit de constatation.

Ce nouveau pouvoir de constatation autorise l'administration à se faire communiquer certains documents en suivant une certaine procédure. Nous touchons ici à la rationalité du contrôle fiscal où l'administration exerce sa compétence de contrôle selon une démarche critique ou contradictoire, c'est le contrôle par communication des tiers.

Ainsi, le pouvoir d'investigation et le pouvoir de vérification sont les deux manifestations du pouvoir de contrôle de l'administration. Il faut y voir deux degrés dans l'investigation fiscale. En nous limitant ici aux pouvoirs d'investigation de l'administration, nous tenterons dans unepremière approche d'examiner les droits $\mathrm{du}$ contribuable face au pouvoir de communication de l'administration, avant de présenter, en deuxième analyse, les droits du contribuable face au pouvoir de constatation de l'administration.

\section{Les droits du contribuable face au pouvoir exercé par l'administration en matière de droit de communication}

L'administration dispose, conformément aux dispositions des articles 46 à 49 et 51 à 52 de la loi $\mathrm{n}^{\circ} 004 / 2003 \mathrm{du} 13$ mars 2003 telle que modifiée et complétée a ce jour portant réforme et procédures fiscales, de la faculté d'exercer son droit de communication auprès de tiers avant, pendant ou après une vérification de comptabilité.

Toutefois, si aucun texte ne s'oppose à ce que l'administration utilise des renseignements provenant d'autres sources que la vérification de comptabilité pour déterminer les bases d'imposition, c'est à la condition que le contribuable en soit informé par le service des impôts et soit mis à même de les contester. Il convient 
de préciser alors la portée de cette garantie liée à l'obligation faite à l'administration d'informer le contribuable de la nature et de la teneur des renseignements recueillis dans l'exercice du droit de communication, mais auparavant nous nous proposons d'examiner l'étendue du pouvoir de communication de l'administration.

Les articles 46 à 49 et 51 à 52 précité et même l'article 53 en définissant le domaine du droit de communication précise les personnes assujetties au pouvoir de communication de l'administration, mais encore les documents sur lesquels il porte. Les entreprises privées sont soumises au premier chef au droit de communication conformément à ce texte.

\section{Les droits du contribuable face au droit d'enquête, de visite et de saisie par l'administration}

Les agents des impôts, munis d'un ordre de missions, peuvent se faire présenter et prendre copies des factures ainsi que des livres, registres et documents professionnels pouvant se rapporter à des opérations ayant donné lieu ou devant donner lieu à facturation.

Ils peuvent également se faire présenter et prendre copies de tous les documents douaniers justifiant la perception de la taxe sur la valeur ajoutée à l'importation, la réalité d'une exportation ou l'application d'un régime suspensif. A l'exception des locaux affectés au domicile privé, ils peuvent, à cet effet, avoir accès, durant les heures d'activités professionnelle, aux locaux à usage professionnel, aux terrains, aux entrepôts, aux moyens de transport à usage professionnel et à leur changement, et procéder à la constations matérielle des éléments physiques de l'exploitation.

Ils peuvent recueillir sur place, des renseignements ou justifications relatifs aux opérations ci-dessus. Ils peuvent, s'il échet, procéder à l'audition du contribuable ou de toute personne afin d'obtenir des renseignements ou des justifications sur la facture reçue ou émise par l'entreprise.

\section{Les droits du contribuable face au pouvoir de vérification et de redressement de la comptabilité par l'administration}

Le passage au système déclaratif a rendu possible la mise en œuvre par l'administration d'un droit de contrôle-(loi n004/2003 du 13 mars 2003 portant reforme des procédures fiscales titre II contrôle telle que modifiée et complétée a ce jour).

Ce droit de contrôle recouvre en pratique un ensemble de pouvoirs de l'administration allant du simple contrôle formel à l'examen contradictoire de la situation fiscale personnelle, en passant par le contrôle sur pièces et la vérification de comptabilité-(article 25 loi $\mathrm{n}^{\circ} 004 / 2003$ du 13 mars 2003 portant reforme des procédures fiscales titre II contrôle, telle que modifiée et complétée a ce jour).

La vérification de comptabilité se distingue néanmoins des autres types de contrôle essentiellement pour trois motifs : en raison d'une part, du lieu où elle est mise en œuvre d'autre part, de son caractère limité quant aux contribuables concernés enfin, de l'étendue des opérations de contrôle.

$\mathrm{Au}$ plan de l'étendue et de l'objet des investigations réalisées, la vérification de comptabilité ne se traduit pas seulement par des opérations matérielles de constatation et de contrôle des documents comptables, elle oblige les vérificateurs, dûment commissionnés à cet égard, à une recherche critique de conformité ou de cohérence des documents comptables et à un examen de régularité et de normalité des actes de gestion des entreprises assujetties.

Toutefois, le législateur a cherché à encadrer ce pouvoir de contrôle, notamment en accordant aux contribuables vérifiés des garanties spécifiques qui délimitent les prérogatives administratives de contrôle. Pour la distinguer alors des autres investigations comptables qui échappent à ces contraintes, la notion de vérification a dû par conséquent être définie et son régime précisé.

Notons ici que le contrôle sur pièces est généralement défini comme étant un contrôle de cohérence entre les différentes déclarations d'un même contribuable d'une part, les informations en provenance de tiers d'autre part, éventuellement complétées par des réponses faisant suite aux demandes de renseignements adressées au contribuable lui même. C'est l'instrument qui permet à l'administration de tirer les premières conclusions quant à l'opportunité d'un contrôle fiscal au titre d'un dossier.

Comme nous l'avons vu, lorsque le contrôle sur place a permis de constater des omissions, des insuffisances ou des dissimulations, le paiement des suppléments de droits exigibles assortis de pénalités ne peut être réclamé en principe qu'après l'aboutissement d'une procédure de redressement.

A cet égard, la procédure de redressement contradictoire prévue à l'article 36 et 37 du Code des impôts livre deuxième est organisée autour de cette formalité substantielle, à savoir la notification de redressement qui est en effet obligatoire.

Il importe de constater que pendant la phase de redressement, les garanties inhérentes au principe du contradictoire sont conditionnées par la procédure mise en œuvre. Lors d'une procédure contradictoire de redressement, le contribuable dispose de différentes opportunités pour faire valoir ses observations. En revanche, dans le cadre d'une procédure d'imposition 
d'office, le principe du contradictoire est presque inexistant.

Après avoir rappelé le champ d'application de la procédure de redressement contradictoire, nous nous proposons d'examiner les garanties attachées à cette procédure.

\section{Les droits du contribuable face au pouvoir de sanctions du Fisc}

L'administration tient de la loi le pouvoir d'appliquer des sanctions qui prolongent ses pouvoirs de contrôle dans le cadre du système déclaratif.

Ces sanctions dites fiscales qui sont essentiellement pécuniaires n'ont pas toujours le même objet. Tantôt, elles visent simplement à assurer le respect d'une obligation imposée au contribuable notamment déclarative et à réprimer alors des manquements à une obligation formelle. Tantôt, elles tendent à punir le contribuable fautif qui a cherché à se soustraire à ses obligations fiscales et qui se rend alors responsable d'une infraction à la loi fiscale.

Comme il importe de constater ici que ces sanctions ne produisent pas toujours les mêmes effets, elles peuvent soumettre en effet le contribuable fautif à un traitement fiscal plus contraignant et se traduire alors par une pénalité distincte de l'imposition. Au demeurant, ces sanctions à « coloration pénale » sont appliquées immédiatement par l'administration conformément à la loi et relèvent de ses pouvoirs.

La jurisprudence a été amenée à admettre ce caractère lorsqu'elle précise que ces sanctions « consistent dans une majoration d'impôt appliquée par l'administration et qu'elles ne peuvent être assimilées aux amendes prononcées par le juge de répression ». L'exercice de ce pouvoir par l'administration qui aboutit en définitive à aggraver la charge fiscale du contribuable s'analyse juridiquement comme une modalité spéciale de l'assiette qui exprime alors une obligation fiscale.

A cet égard, il importe de constater que l'administration, pour reprendre la formule du Professeur L. TROTABAS, « est plus libre pour appliquer les sanctions que pour appliquer l'impôt, car celui-ci suppose une compétence liée, tandis que celleslà présentent un caractère discrétionnaire »-(L. TROTABAS, JM. COTTERET, 1977).

\section{CONCLUSION}

$\mathrm{Au}$ terme de notre étude, après observation et analyse, il sied de relever certains faits consécutifs à l'exécution du contrôle fiscale appliqué par l'administration fiscale de la RDC. Tout d'abord l'intervention du législateur notamment par la loi-cadre n $004 / 2003$ du 13 mars 2003 telle que modifiée et complétée a ce jour relative à la réforme des procédures fiscales en faisant une large place au régime déclaratif a renforcé d'autant les procédures administratives de contrôle fiscal.

Mais, ce renforcement des moyens juridiques de l'administration de contrôle s'est accompagné de procédures en matière d'investigations fiscales dites « périphériques » qui bénéficient de bien moins de garanties et qui présentent de surcroît des risques de détournements de procédure.

Ainsi, les contrôles matériels inopinés que représente le droit de constatation se rattachent légalement à la vérification de comptabilité. Les risques de détournement de cette procédure de contrôle ne se posent pas en principe, puisque les constatations matérielles sont formellement intégrées dans la vérification de comptabilité, mais en réalité des risques existent.

En ce sens, les constatations matérielles sont effectuées dans le cadre des dispositions de l'article 25 du code des impôts livre deuxième titre II: le contribuable est alors informé dès le début des opérations de contrôle inopiné suivant l'avis de constatation qu'on lui remet séance tenante, mais les enquêteurs débutent immédiatement leurs constatations matérielles débordant sur une vérification de comptabilité « innomée » excluant ainsi le contribuable de l'avantage des garanties spécifiques attachées à cette procédure.

Nous nous interrogeons alors dans ces conditions si l'administration en mettant à profit l'article 25 du Code des impôts ne cherche pas à contourner l'interdiction qui lui est faite d'effectuer des contrôles inopinés au fond?

En outre, le droit de communication est une procédure d'investigation distincte $\mathrm{du}$ droit de vérification de comptabilité au regard du régime légal qui l'organise.

Toutefois, en pratique il présente un risque élevé de détournement de procédure. Ainsi, comme la vérification de comptabilité, il permet à l'administration d'exercer son droit à l'information notamment en se rendant sur place chez le contribuable.

Celle-ci peut alors être tentée d'effectuer une exploitation critique des documents consultés sur place au mépris des droits de la défense. Du reste, le foisonnement des moyens désencadrés du contrôle fiscal renforce les possibilités de mise en œuvre de vérifications occultes de comptabilité fragilisant d'autant l'édifice des opérations de vérification de comptabilité et celui des garanties du contribuable qui en sont les précieuses opinions. 
En définitive, l'administration fiscale doit pouvoir réaliser le contrôle fiscal dans le stricte respect des procédures fiscales, prévues dans la loi n004/2003 du 13 mars 2003 telle que modifiée et complétée à ce jour, de façon à garantir un prélèvement juste de l'impôt et aussi d'entretenir des bonnes relations avec les contribuables. Etant donné que ces derniers doivent être considérés comme des partenaires et non des esclaves fiscales.

\section{Ainsi, nous pouvons affirmer ce qui suit}

- Une administration qui vous simplifie la vie trouvera en face un contribuable citoyen ;

- Une administration qui respecte les personnes et le droit trouvera en face un contribuable coopératif ;

- Une administration équitable trouvera en face un contribuable loyal.

\section{REFFERENCE}

1. DGI : code des impôts mis à jour au 30/09/2003 telle que modifiée et complétée a ce jour;
2. Manuel des procédures fiscales de novembre 2004 dgi rdc ayaye fafay

3. Guide de contrôle fiscal des entreprises commerciales et industrielles, CRIGED

4. Manuel des procédures fiscales de novembre 2004 DGI RDC

5. Loi $n^{\circ} 004 / 2003$ du 13 mars 2003 portant reforme des procédures fiscales telle que modifiée et complétée a ce jour ;

6. Loi $\mathrm{n}^{\circ} 13 / 005$ modifiant et complétant certaines dispositions de la loi $\mathrm{n}^{\circ}$ 004/2003 du 13 mars 2003 portant réformes et procédure fiscales.

7. Bouvier M. Introduction au droit fiscal général et à la théorie de l'impôt, LGDJ, 7è

8. Verges E. Procédure civile, PUG, 2007édition, 1977

9. Trotabas L. Finances publiques, Dalloz, 4e édition, 1971

10. Trotabas L. Cotteret JM., droit fiscal, Paris, Précis Dalloz, 3ème. 